\title{
La investigación científica expuesta a estudiantes de grado: un caso práctico de Física de la Materia Condensada Exposing undergraduate students to research: A Condensed Matter Physics case study
}

\author{
Yuriko Baba ${ }^{1}$, Elena Díaz ${ }^{1}$, Francisco Domínguez-Adame ${ }^{1}$, Álvaro Díaz-Fernández ${ }^{2}$ \\ yuribaba@ucm.es, elenadg@ucm.es, adame@ucm.es, alvaro.diaz@upm.es \\ ${ }^{1}$ Departamento de Física de Materiales \\ Universidad Complutense de Madrid \\ Madrid, España \\ ${ }^{2}$ Departamento de Estructuras y Física de Edificación \\ Universidad Politécnica de Madrid \\ Madrid, España
}

\begin{abstract}
Resumen- En la actualidad, la información tiende a transmitirse de un modo muy sensacionalista y superficial. En especial, en el caso de la divulgación de los avances científicos, los medios tienden a presentar descripciones superficiales y sesgadas hacia temáticas que puedan parecer más llamativas. Esta praxis es particularmente preocupante en cuanto puede influenciar las decisiones de los estudiantes que quieren emprender una carrera investigadora. Más aún, los programas de estudio no dejan espacio a introducir conceptos que vayan más allá del currículo académico, imposibilitando que se pueda paliar esta problemática en el aula. Con el objetivo de hacer frente a esto, hemos organizado un taller donde expertos de diversas instituciones impartieron conferencias y realizaron experimentos relacionados con nuevos descubrimientos en la Materia Condensada, la rama de la física que estudia las propiedades de sistemas cuánticos de muchos cuerpos en interacción. La iniciativa, denominada Últimos Avances en Física de la Materia Condensada, estaba dirigida a estudiantes de Grado en Física en sus dos últimos años en nuestra Universidad. Con el fin de evaluar nuestra metodología, el taller se llevó a cabo durante tres años académicos consecutivos y se recogieron las opiniones de los asistentes en forma de tareas y encuestas. Los resultados muestran que la introducción de investigaciones recientes en los planes de estudio es una estrategia compleja pero fructífera.
\end{abstract}

Palabras clave: Metodologías de aprendizaje; iniciación de pregrado en investigación; orientación estudiantil; física de Materia Condensada

Abstract- Currently, the media tends to present the news sensationally and superficially. In an effort to communicate major scientific breakthroughs to a vast audience, the media presents brief accounts of the findings and more emphasis will be put on those discoveries with more captivating titles. This is particularly worrying when it comes to actual students as it utterly biases their decisions when aiming to pursue a research career. The fact that syllabi tend to leave little to no room for introducing concepts that go beyond the standard curriculum leaves this problem unsolved. With the aim of tackling these issues, we have organized a workshop where experts from various institutions delivered lectures and even performed exhibitions of the phenomena related with Condensed Matter Physics, the field of physics that deals with properties of many-body quantum system with interactions. The initiative, named Recent Advances in Condensed Matter Physics, was aimed at undergraduate Physics students in their last two years at our University. In order to assess the strategies of our learning methodology, the experiment was carried out for three consecutive academic years and feedback from students was collected in the form of homework and surveys. The results show that introducing recent research discoveries in the curricula is a complex yet profitable strategy.

Keywords: Learning methodologies; undergraduate initiation in research; student guidance; condensed matter physics.

\section{INTRODUCCIÓN}

El término Física de la Materia Condensada (FMC) fue acuñado por Philip Warren Anderson (Premio Nobel de Física en 1977) y Volker Heine en 1967, cuando trabajaban en el Laboratorio Cavendish de Cambridge. Esta área se ocupa de las fases condensadas de la materia, a saber, sólidos y líquidos con muchos componentes (electrones y iones) bajo fuertes interacciones entre ellos y posiblemente sometidos a campos electromagnéticos externos. Según el número de artículos publicados, la FMC es el sub-campo más activo de investigación en Física hoy en día (Sinatra y col. 2015). Desde el año 2000, la Real Academia Sueca de Ciencias ha otorgado el Premio Nobel de Física seis veces a los investigadores que trabajan en áreas relacionadas con FMC, incluyendo la última en 2016. Además del interés por aspectos fundamentales de la Física, la FMC es un área clave para el desarrollo de nuevas tecnologías en una variedad de nichos (tecnologías cuánticas y de la información, almacenamiento de datos, recolección de energía y materiales a medida para una serie interminable de aplicaciones, por nombrar solo algunas). Un gran número de científicos formados en FMC trabajan en la industria y encontraron la formación que recibieron en la Universidad muy gratificante. Un ejemplo paradigmático es uno de los propios padres fundadores de la FMC, Philip W. Anderson, quien recibió el Premio Nobel siendo empleado de Bell Telephone Laboratories (Anderson, 2021).

La gran mayoría de los grados de Física, si no todos ellos, ofrecen materias relacionadas con la FMC, desde los niveles introductorios (en su mayoría conocidos como cursos de Física de Estado Sólido) hasta los avanzados. Sin embargo, los programas de estas asignaturas son a menudo extremadamente densos y amplios, con una gran variedad de temas a tratar. 
Como resultado, por lo general hay muy poco tiempo para discutir descubrimientos científicos recientes. Tales descubrimientos a menudo se explican brevemente en los medios de comunicación y a veces incluso pueden pasar desapercibidos por no ser lo suficientemente cautivadores. Un ejemplo significativo de esta tendencia es la comparación de la repercusión mediática de observaciones recientes en distintos campos de la física. La primera fotografía de un agujero negro realizada por el Event Horizon Telescope en 2019 ha generado del orden de 18900 noticias (número de resultados de Google Noticias al buscar "Event Horizon Telescope black hole") mientras que el descubrimiento de fases super- conductoras en grafeno por el grupo de Pablo Jarillo-Herrero ronda los 1050 artículos (búsqueda: “Twisted bilayer graphene"). Esta asincronía entre el contenido de los planes de estudios estándar y los descubrimientos de vanguardia crea una laguna en el conocimiento de los alumnos de grado, que los estudiantes no completan hasta asistir a cursos de posgrado. Para entonces, los estudiantes ya han elegido un camino a seguir en su investigación sin haber tenido suficiente información sobre hallazgos novedosos en diferentes campos de las ciencias físicas.

A lo largo de los años, hemos podido observar cómo los estudiantes perciben la mencionada asincronía entre los conceptos que se están introduciendo en sus clases y la investigación actual en campos tales como la FMC. Un excepcional ejemplo es el grafeno, un material que, desde su descubrimiento experimental en 2010, ha liderado una plétora de descubrimientos revolucionarios. Por el contrario, los estudiantes de cursos de grado aprenderán que el grafeno es un material cristalino con propiedades interesantes sin profundizar más en ellas. Una excelente revisión sobre cómo cerrar la brecha entre la enseñanza y la investigación se puede encontrar en Fraser $y$ col. (2014). Con esto en mente, decidimos poner a prueba la idea de acercar los recientes avances en FMC a los estudiantes, relacionando los aspectos básicos presentados en las asignaturas tradicionales con la investigación más actual, además de explicar cómo ésta ha remodelado nuestro mundo moderno. En este trabajo presentaremos las iniciativas y actividades que se llevaron a cabo durante los cursos académicos 2017/18, 2018/19 y 2019/20, junto con los principales resultados y conclusiones que obtuvimos.

\section{CONTEXTO}

\subsection{Objetivos}

Este trabajo se cimenta en torno al taller Últimos Avances en Física de la Materia Condensada (UAFMC). Todas las actividades y la recopilación de datos provienen del susodicho taller. Más específicamente, nuestros objetivos al diseñar este proyecto docente han sido:

- Familiarizar a los estudiantes con los temas de investigación más recientes y destacados en el área, con el fin de elevar el interés de los estudiantes en la FMC.

- Aumentar el interés en iniciar una carrera de investigación y, en particular, llamar la atención de los estudiantes sobre la FMC.
- Evaluar estadísticamente la opinión de los estudiantes mediante encuestas. Nuestro objetivo era averiguar si la asistencia al taller aumentaba su conciencia sobre la importancia de los temas relacionados con la FMC dentro de su formación.

- Mostrar los resultados y conclusiones a las autoridades académicas de nuestro Departamento, con el objetivo de introducir nuevas metodologías en el aula.

Pese a que este estudio está centrado en el caso particular de la FMC, estos mismos objetivos se pueden trasladar a cualquier rama de conocimiento específica y delimitada a la que se quiera dar visibilidad desde una perspectiva investigadora.

\subsection{Impacto e indicadores esperados}

El impacto esperado es muy específico y fácilmente cuantificable. Por un lado, hay una serie de aspectos que se discutirán en detalle más adelante en este trabajo. Estos son la organización del taller, los seminarios, el número de participantes, el número de créditos ECTS opcionales, los ponentes, etc., algunos de los cuales ya proporcionan indicadores relacionados con las muestras estadísticas que se han utilizado para las encuestas. Por otro lado, tenemos las encuestas cuyas respuestas se encuentran en la sección 4. Por último, en esta misma sección se incluye un Libro Blanco con propuestas específicas elevadas a las autoridades académicas de nuestro Departamento. Esperamos que el impacto positivo sea inmediato, ya que las actualizaciones en los contenidos y las nuevas metodologías son fácilmente accesibles para su introducción en el plan de estudios actual.

\section{DESCRIPCIÓN}

\subsection{Organización}

El taller UAFMC se llevó a cabo durante el segundo semestre de cada curso académico, de febrero a mayo, durante los cursos académicos 2017/18, 2018/19 y 2019/20. La audiencia eran estudiantes de tercer y cuarto año del Grado en Física. Asimismo, se han aceptado las solicitudes de alumnos de Máster dado que, pese a haber elegido ya el área de especialización, se ha considerado que pudieran beneficiarse de adquirir una perspectiva más amplia dentro de la FMC. Cada sesión duró 90 minutos y tuvo lugar una vez a la semana, a lo largo de 10 semanas lectivas. Encontrar el momento adecuado para las conferencias no fue sencillo, ya que el alumnado de los últimos cursos tienen una amplia variedad de asignaturas optativas que se superponen frecuentemente. Cada sesión fue impartida por un experto en FMC, tanto del mundo académico como de laboratorios de investigación e instituciones científicas (véase la Figura 1). Todos los investigadores contactados para las charlas aceptaron la invitación, mostrando de este modo gran interés en iniciativas de esta índole. 


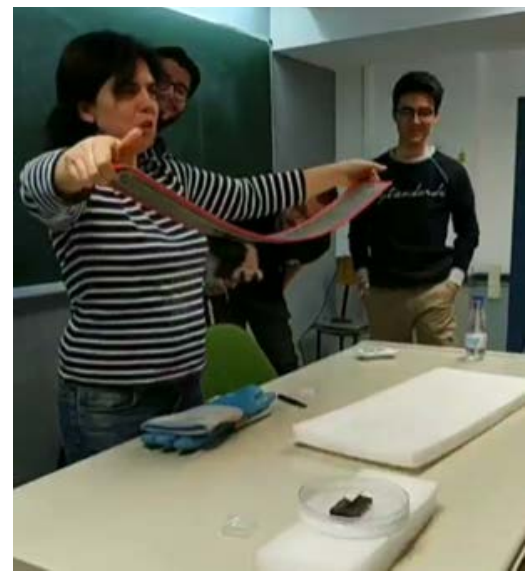

Figura 1. La profesora María José Calderón, del Instituto de Ciencia de Materiales de Madrid, en una demostración en el aula de la increíble física de los superconductores de alta temperatura crítica.

El número de estudiantes que asistieron al taller cada año académico fue superior a 60 , mucho más alto que los alumnos matriculados en las asignaturas optativas de FMC. La mayoría de los estudiantes asistieron a los seminarios regularmente y recibieron un diploma de participación y asistencia. A los estudiantes también se les dio la posibilidad de entregar un resumen de aproximadamente 1000 palabras relacionadas con una de las sesiones de su propia elección. Aquellos que lo hicieron pudieron solicitar 1 crédito ECTS de libre configuración.

\subsection{Temas}

A modo de ejemplo, se recogen a continuación los títulos de las sesiones del curso académico 2019/20

(1) Moléculas quirales: Espintrónica sin imanes.

(2) Aisladores topológicos y semimetales topológicos.

(3) Los efectos cuánticos de Hall.

(4) Grafeno bicapa retorcido: la magia de Moiré.

(5) Propiedades ópticas de nanohilos semiconductores.

(6) Puntos cuánticos: una plataforma versátil hacia el cálculo cuántico y la simulación cuántica.

(7) Fortalezas y debilidades de los cálculos ab-initio.

(8) Nanohilos: una ruta hacia dispositivos termoeléctricos eficientes.

(9) Superconductividad.

(10) Transporte térmico a nanoescala.

La selección se ha realizado atendiendo al criterio de maximizar la diversidad de temas dentro de la FMC.

Las sesiones incluían contenidos tanto de carácter teórico como práctico. Por ejemplo, tomando como referencia las charlas del último año académico impartido 2019/2020, exactamente la mitad de los ponentes fueron investigadores de ramas teóricas y mitad de enfoques experimentales.

En cuanto a las sesiones, se incluyeron elementos variados, desde contenidos o experimentos introductorios de carácter divulgativo hasta resultados experimentales o simulaciones a nivel investigador. Se trata, por lo tanto, de métodos de enseñanza que se acercan más a un modelo de aprendizaje activo que a una clase magistral, como los propuestos por Fraser y col. (2014).

Debido a la crisis del Covid-19, las últimas cinco sesiones del curso 2019/20 se impartieron en línea.

\subsection{Preguntas de la encuesta}

Al final del taller, los estudiantes respondieron a una encuesta anónima que contenía las siguientes preguntas:

(1) ¿Has estado o estás matriculado en asignaturas de física de Materia Condensada en tus estudios de grado o máster?

(2) ¿Conocías lo que era la física de la Materia Condensada antes de asistir al taller?

(3) Después de este taller, ¿ha aumentado tu interés en profundizar en los conocimientos a través de otros cursos, actividades o asignaturas optativas sobre temas relacionados con la física de la Materia Condensada?

(4) ¿Te gustaría iniciar la carrera investigadora en física de Materia Condensada?

(5) Si es así, ¿qué aspectos te interesan más? Teórico, experimental o ambos.

(6) ¿Cree que el número de asignaturas que se ofrecen sobre física de la Materia Condensada es acorde con su relevancia?

(7) ¿Cree que el formato del taller, con charlas específicas sobre temas de investigación actuales, es interesante y aplicable a otras asignaturas de grado?

(8) ¿Estás considerando solicitar el crédito opcional?

(9) Si es así, ¿crees que el método de evaluación es apropiado?

(10) ¿Volverías a asistir al taller el próximo año si se presentan nuevos temas?

(11) ¿Recomendarías a tus compañeros de clase la asistencia al taller?

(12) ¿Qué temas echaste de menos en el taller de este año?

Datos de las estadísticas: Hombre Mujer

\section{RESULTADOS}

4.1 Datos estadísticos

La Tabla 1 presenta un resumen de los datos obtenidos mediante las encuestas. 
Tabla 1. Estadísticas de respuestas a las preguntas de la encuesta.

\begin{tabular}{|c|c|c|c|}
\hline Pregunta & Sí & No & NS/NC \\
\hline 1 & $18,5 \%$ & $81,5 \%$ & 0 \\
\hline 2 & $70,4 \%$ & $29,6 \%$ & 0 \\
\hline 3 & $88,9 \%$ & $11,1 \%$ & 0 \\
\hline 4 & $48,2 \%$ & $40,8 \%$ & $11,0 \%$ \\
\hline 6 & $40,7 \%$ & $59,3 \%$ & 0 \\
\hline 7 & $92,6 \%$ & $7,4 \%$ & 0 \\
\hline 8 & $18,5 \%$ & $81,6 \%$ & 0 \\
\hline 9 & $100,0 \%$ & 0 & 0 \\
\hline 10 & $92,6 \%$ & $7,4 \%$ & 0 \\
\hline 11 & $96,3 \%$ & 0 & $3,7 \%$ \\
\hline
\end{tabular}

La pregunta 5 era una pregunta continuación de la pregunta 4 que, en caso de que ésta fuera afirmativa, pretendía averiguar el interés del estudiante en aspectos teóricos o experimentales en FMC. Las respuestas fueron las siguientes

\begin{tabular}{|c|c|c|c|}
\hline Teoría & Experimento & Ambos & NS/NC \\
\hline $14,8 \%$ & $33,3 \%$ & $29,6 \%$ & $22,3 \%$ \\
\hline
\end{tabular}

\subsection{Género del alumnado encuestado}

Los datos utilizados para la Figura 2 corresponden al curso académico 2018/19.

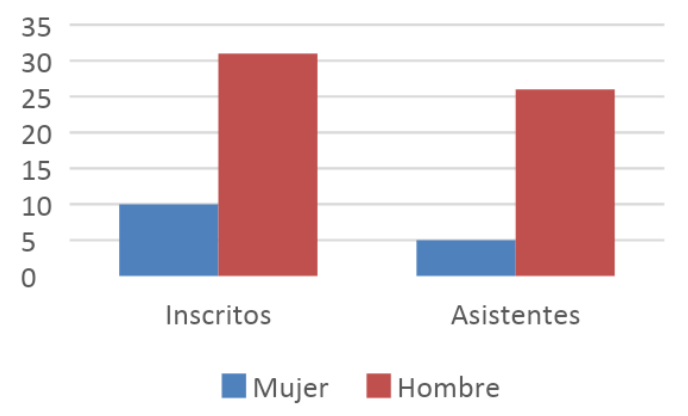

Figura 2. Género de inscritos y asistentes al taller UAFMC, encuestados en 2018/19.

La proporción de participación por género responde a la media de $36 \%$ de mujeres de nuestra facultad (Nieto-Zayas y col. 2013).

\subsection{Análisis de los resultados}

A partir de los resultados obtenidos en las preguntas 1 y 2 , con respecto a su conocimiento de la FMC y haber cursado alguna asignatura relacionada antes de los seminarios, la mayoría de los estudiantes respondieron que sabían de la existencia de la FMC aunque aún no se habían matriculado en ninguna asignatura relacionada con ella. Esto es muy probablemente debido al hecho de que la mayoría de los estudiantes saben que el programa de la asignatura de Física del Estado Sólido, que es troncal, abarca temas que pertenecen al área de FMC. La pregunta 3 muestra que el taller ha cumplido nuestro objetivo de elevar el interés de los participantes en los aspectos científicos relacionados con la FMC, ya que la mayoría de los estudiantes muestran interés en seguir profundizando sobre los temas presentados. Además, la mitad de los asistentes considera la posibilidad de iniciar la carrera científica en el campo de la FMC (pregunta 4), en una proporción que es incluso mayor que la proporción de físicos que realmente trabajan en FMC. El interés por los aspectos puramente teóricos es bajo, alrededor de un 15\% (pregunta 5) $\mathrm{y}$, notablemente, todos los participantes interesados eran hombres. Por el contrario, el $100 \%$ de las encuestas que se decantaron por los aspectos puramente experimentales correspondía a mujeres. Sin embargo, es interesante señalar que alrededor de un tercio de los participantes desearían seguir una carrera mixta, ni puramente teórica ni puramente experimental. Como muestra la pregunta 6 , una gran proporción de los estudiantes, alrededor del $60 \%$, considera que la FMC está infrarrepresentada en el currículo actual. Las respuestas a la pregunta 7 muestran que la mayoría de los estudiantes creen que este tipo de taller se puede extender a otras materias y asignaturas. Aunque solo un pequeño número de estudiantes deseaban solicitar el crédito de libre elección (pregunta 8), todos ellos consideraron apropiado el método de evaluación (pregunta 9). Por último, nos gustaría destacar el gran número de estudiantes (por encima del 90\%) que deseaban asistir al taller del año siguiente y lo recomendarían a sus compañeros de clase (preguntas 10 y 11). En cuanto a la actividad realizada para solicitar el crédito optativo, se consideró como calificaciones las de apto y no apto, atendiendo a criterios de comprensión y corrección de los fenómenos físicos descritos.

Por último, es interesante notar cómo el número de matriculados en la asignatura optativa de Física de la Materia Condensada se ha visto acrecentado en los años académicos en los que se llevó a cabo el taller, como muestra la Figura 3. Consideramos este aumento como un indicador adicional del cumplimiento de los objetivos propuestos. Por otro lado, Creemos que la bajada en el número de matriculados en el curso 2020/2021 no es un dato significativo por las circunstancias excepcionales que han afectado a este curso académico dado los protocolos de docencia semipresencial por la pandemia COVID. 


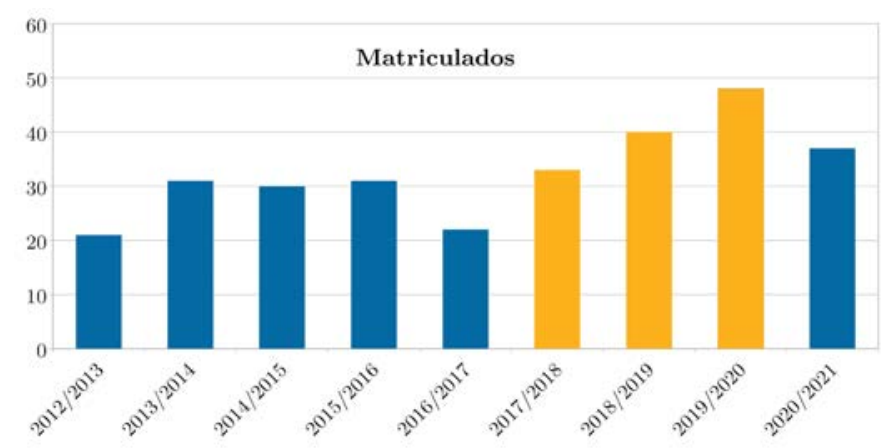

Figura 3. Evolución del número de matriculados en la asignatura optativa de Física de la Materia Condensada. En naranja se señalan los años coincidentes con el taller UAFMC.

\subsection{Libro blanco}

A partir de los resultados obtenidos en las encuestas, así como de la experiencia adquirida por los organizadores, hicimos las siguientes recomendaciones a nuestro Departamento:

- En las primeras asignaturas del Grado en Física donde los estudiantes comienzan analizar el comportamiento a nivel atómico de la materia sólida, a saber, física de materiales y física de estado sólido, es conveniente recalcar que la FMC representa un tercio de la investigación actual en física moderna.

- Dado que las verdaderas modificaciones de los programas podrían llegar a ser bastante complejas, se deben plantear alternativas para mostrar a los estudiantes la relevancia de la FMC. El taller UAFMC demostró ser una excelente plataforma para lograr este objetivo, aunque claramente no es la única alternativa que existe.

- Ofrecer créditos opcionales favorece la participación, incluso si el estudiante ya tenía una motivación previa para asistir a la actividad.

- El taller ha permitido el análisis, síntesis y evaluación de los contenidos en FMC desde el punto de vista de los estudiantes.

\section{CONCLUSIONES}

Las encuestas muestran que el taller UAFMC resultó ser una actividad interesante para que los estudiantes conozcan la investigación de vanguardia de la Física y aumenten su interés en la materia. Los resultados y el análisis de los mismos han sido incluidos en un Libro Blanco que se presentó a las autoridades de nuestro Departamento. El resultado esperado es que las mejoras se irán incluyendo paulatinamente en las metodologías de enseñanza, así como en los contenidos de diversas asignaturas.

Los resultados del taller de UAFMC parecen responder afirmativamente a la pregunta de Fraser y col. (2014): ¿Los estudiantes tienen tiempo y disposición para una enseñanza basada en la investigación? Por ello, teniendo en cuenta el interés de los estudiantes y la necesidad de mejorar la apreciación que tienen sobre la relevancia actual de la FMC, un hecho que no parece exclusivo de nuestra Universidad, creemos que los resultados aquí presentados serán de interés para otras Universidades y para otros campos de estudios.

\section{AGRADECIMIENTOS}

Los autores agradecen a Dunkan Martínez por sus útiles discusiones. Este trabajo ha sido apoyado por el Ministerio de Ciencia e Innovación (Grant PID2019-106820RB-C21).

\section{REFERERENCIAS}

Philip W. Anderson. Nobel Prize Outreach (2021). https://www.nobelprize.org/prizes/physics/1977/anderson/facts

Fraser, J. M., Timan, A. N., Miller, K., Dowd, J. E., Tucker, L., \& Mazur, E. (2014). Teaching and physics education research: bridging the gap. Rep. Prog. Phys. 77, 032401. doi: 10.1088/0034-4885/77/3/032401.

Nieto-Zayas, C. y col. (2013). Estudio sobre la inserción laboral de los licenciados en Física Promociones de los años 2009, 2010 y 2011. Universidad Complutense de Madrid. https://www.ucm.es/data/cont/docs/3-2014-12-04F\%C3\%8DSICA.\%202013.pdf .

Sinatra, R., Deville, P., Szell, M., Wang, D., \& Barábasi, A. L. (2015). A century of physics. Nat. Phys. 11, 791-795. doi: $10.1038 /$ nphys 3494 . 